\title{
INFLUENCE OF SUBSTRATE THICKNESS ON DIFFUSE COPLANAR SURFACE BARRIER DISCHARGE PROPERTIES
}

\author{
Jan Čech*, Miroslav Zemánek, Pavel SŤahel, Hana Dvořáková, \\ MIRKO ČERNÁK
}

\author{
CEPLANT - REDD Centre For Low-cost Plasma And Nanotechnology Surface Modifications, Masaryk \\ University, Kotlářská2, 61137 Brno, Czech Republic \\ * corresponding author: cech@physics.muni.cz
}

\begin{abstract}
This paper presents an investigation of the influence of dielectric barrier thickness on the parameters of the Diffuse Coplanar Surface Barrier Discharge. The discharge was operated in an ambient air at atmospheric pressure. The electrical parameters of the system were studied both experimentally and using numerical simulations. The discharge pattern was also studied using an intensified CCD camera.
\end{abstract}

KEYwORDS: DCSBD, coplanar discharge, dielectric, thickness, ICCD, electric field simulation.

\section{INTRODUCTION}

Dielectric barrier discharges (DBDs) have been known to the scientific community for more than 150 years [1, 2]. Since their invention, DBDs have been used extensively for ozone generation [1, 3]. The ozone production ratio depends strongly on the gas temperature, as ozone decays considerably with increasing temperature [4. Because of their thermal nonequilibrium nature [5], and since the storage and transportation of ozone poses serious hazards, DBDs have been under continuous investigation for ozone production. However the thermal non-equilibrium nature of DBDs is favourable not only for ozone production, but also for plasma processing of various materials [2], especially thermally sensitive materials, such as polymers, paper, woven and non-woven textiles, etc. 6-10. DBDs have also been tested as tools for plasma medicine [11, 12] or for bio-decontamination (sterilization) of surfaces [13, 14].

The most common type of DBDs used for material plasma processing is so-called the 'industrial corona' [15], i.e., the volume DBD [16. Although their design is very simple and they are cheap to use the properties of the volume DBDs do not meet the industrial demands on plasma processing. Volume DBDs contract in numerous strong and thin microdischarges with increasing power density [17, 18, limiting the contact area with the processed sample and potentially causing local damage to the sample. This is closely connected with limited energy efficiency, since a significant part of the plasma that is created is not in contact with the sample (discharge geometry). Moreover, volume DBDs can practically be generated only in a thin slit or gap because of the high electric field (high voltage) requirement, which strongly limits the thickness of the treated sample to only a few millimeters.

In response to industrial demands a type of planar configuration of the DBDs so-called diffuse coplanar surface barrier discharge (DCSBD) was invented by M. Cernak 19. Plasma of DCSBD is generated in a thin layer above a dielectric $(0.3 \mathrm{~mm}$ at $1 \mathrm{~atm}$ in air [19]), at relatively high power densities $\left(135 \mathrm{~W} \mathrm{~cm}^{-3}\right.$ at $1 \mathrm{~atm}$ in air ${ }^{7}$, while becoming more diffuse with increasing power input. The discharge still consists of thin channels (filaments or micro-discharges) crossing the electrode gap between the electrodes [20], but microdischarges are oriented parallel to the treated sample and they form a visually diffuse-like layer above the electrodes. These properties make DCSBD a promising candidate for high-speed plasma processing of various materials 21.

Because of its application potential, the properties of DCSBD have been under investigation and many industrial applications have been tested in the last decade [8, 22-27].

This paper presents a study of the practical impacts of dielectric barrier thickness on the parameters of DCSBD by means of electrical measurements and highspeed ICCD camera imaging. The dielectric barrier serves firstly as the electric insulation of the electrodes, and it also works as a mechanical holder for the electrode system and as place of contact between the discharge and the treated samples. These functions are in contradiction with each others. The electric parameters induce a requirement for the dielectric thickness to be as low as possible in order to avoid dielectric losses and lower the power supply voltage. However, the mechanical parameters require high mechanical stability of the DCSBD system, which is important for industrial applications of DCSBD plasma systems. The thickness of the dielectric barrier has to be kept above the critical level to prevent the dielectric barrier cracking.

\footnotetext{
${ }^{1}$ Power density of $135 \mathrm{~W} \mathrm{~cm}^{-3}$ is computed from the power input of $650 \mathrm{~W}$ to a volume of $4.8 \mathrm{~cm}^{3}$ of DCSBD plasma.
} 


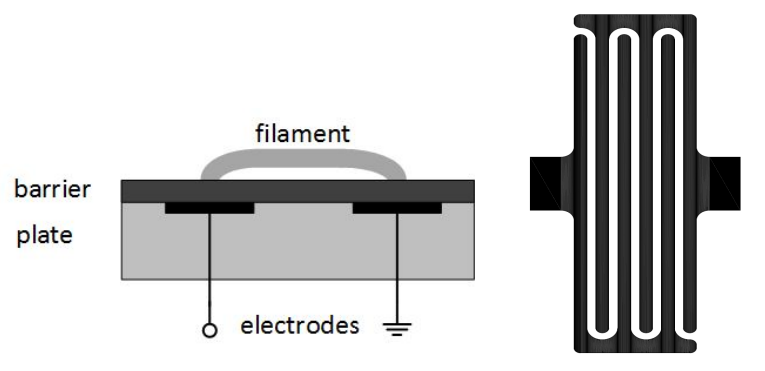

FiguRE 1. DCSBD cell schema: left) discharge cell cross-section; right) ground-plan of the electrode system .

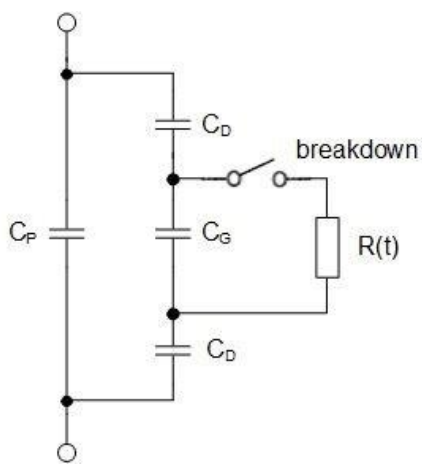

Figure 2. Simple equivalent circuit of the DCSBD.

\section{EXPERIMENTAL SETUP}

A new oil-less DCSBD system is presented here. The discharge cell consists of three electrode pairs embedded in ceramics. The design of the discharge cell does not use an oil cooling system. Instead, a passive heater is utilized. The operation regime is therefore non-continuous with a maximum dissipated power of a few tens of Watts and an operating time of approx. 1 minute and 5 minutes duty time. Due to the unique design, the discharge cell is highly favourable for use in a clean processing environment or in low pressure systems.

Fig. 1 shows the DCSBD discharge cell schema. The glass-ceramics base plate $5.0 \mathrm{~mm}$ in thickness is covered with an electrode system of three electrode pairs $45 \mathrm{~mm}$ in length, $1.5 \mathrm{~mm}$ in width and with a mutual distance (gap) of $1.0 \mathrm{~mm}$. The top (barrier) ceramic plate is made of $96 \%$ alumina ceramics $\left(\mathrm{Al}_{2} \mathrm{O}_{3}\right)$ $0.2 \mathrm{~mm}, 0.6 \mathrm{~mm}$ and $1.0 \mathrm{~mm}$ in thickness respectively. The discharge is operated in free-standing mode in ambient air.

Fig. 2 shows a sketch of the DCSBD simple equivalent circuit. The dielectric glass-ceramic substrate is labeled as the plate. The simple equivalent circuit consists of two parallel connected capacitors - the capacity of the plate $\left(\mathrm{C}_{\mathrm{P}}\right)$ and the serial connected capacities of the dielectric barrier $\left(\mathrm{C}_{\mathrm{D}}\right)$ and the gas $\left(\mathrm{C}_{\mathrm{G}}\right)$. The discharge (filament), which shorts the gas capacitor when there is a discharge breakdown, then works as a time-dependent resistivity.

The experimental setup is shown in Fig. 3. The power to the discharge cell is supplied using a specially

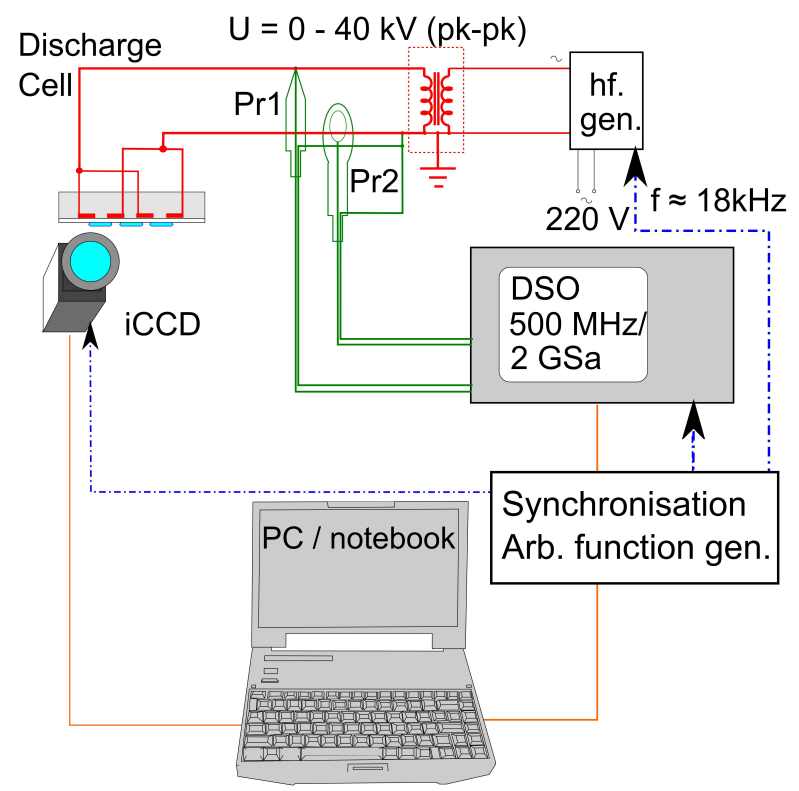

Figure 3. Experimental setup: general scheme of the discharge setup and time-resolved optical imaging.

designed high-voltage (HV) high-frequency power supply. The output voltage is a sine-wave with maximum amplitude of $20 \mathrm{kV}$ and a tunable frequency in the range of approx. 10 to $50 \mathrm{kHz}$. The frequency sourceclock signal for the generator is supplied externally using an arbitrary function generator, see below.

The voltage-current characteristics were recorded using an HP 24820A Infinium 2-channel $500 \mathrm{MHz} / 2 \mathrm{GSa}$ digital storage oscilloscope coupled with HV probe Tektronix P6015A 1000:1 (in Fig. 3 labeled Pr1) and Pearson Current Monitor 2877 (labeled Pr2 in Fig. 3).

For high speed synchronized discharge imaging, the Princeton Instruments PI-MAX3 1024i-SR-46-CM ICCD camera was used. The camera intensifier has sensitivity in the range from 200 to $900 \mathrm{~nm}$ and a minimum gate time of $3 \mathrm{~ns}$. The camera was equipped with a SIGMA lens (105 mm 1:2.8 DG MACRO EX) with true 1:1 macro capability. The ICCD camera was placed along the axis of symmetry perpendicular to the DCSBD plasma layer.

The experimental setup enables us to take phaselocked images of the discharge pattern with respect to the voltage-current waveforms of the discharge. The Agilent 33220A arbitrary function generator was used as the synchronization signal source. The synchronization signal served as the reference internal clock signal for the HV generator, and it served simultaneously as the triggering/gating signal for the ICCD camera.

\section{Results And Discussion}

\subsection{Electrical measurements}

The influence of dielectric barrier thickness on the operating parameters of DCSBD was studied. The dielectric barrier has a principal influence on the DBD 

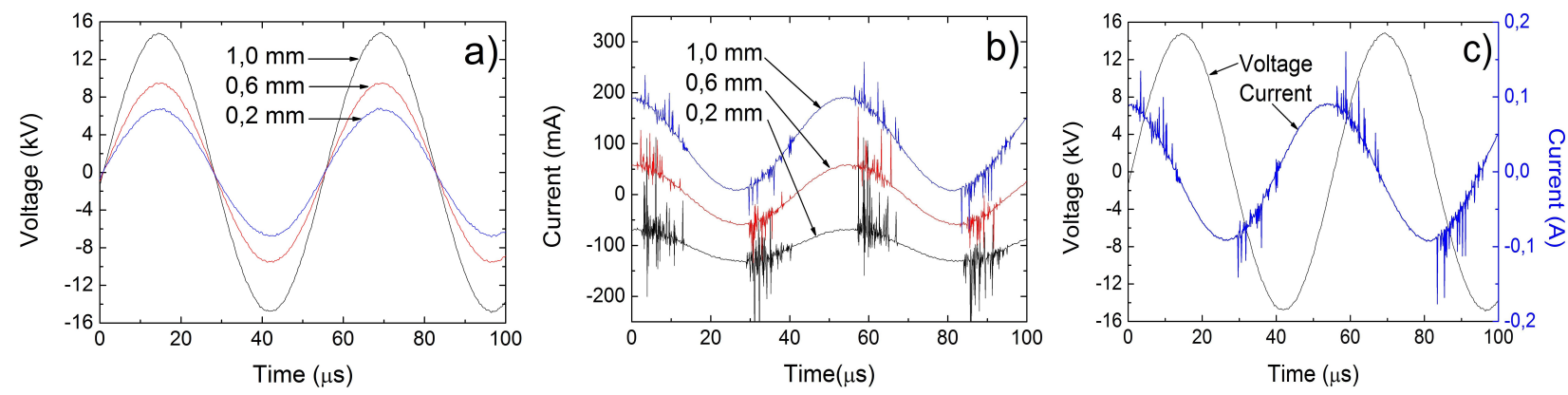

FIGURE 4. The electrical parameters of DCSBD utilizing various alumina barrier thicknesses: a) the voltage waveforms and b) the current waveforms for various barrier thickness, at just full coverage of the electrode surface with plasma; c) the representative current-voltage waveforms for DCSBD utilizing a barrier $1.0 \mathrm{~mm}$ in thickness at a voltage amplitude of $14.9 \mathrm{kV}$ and the frequency of $18 \mathrm{kHz}$.

parameters. The barrier composition and also the barrier thickness were studied for different setups of DBD. In this paper, the influence of barrier thickness was studied for the DCSBD discharge in the new oil-less configuration using a $96 \%$ alumina ceramic plate as the dielectric barrier. The DCSBD parameters, such as operational voltage, voltage-current waveforms and the pattern of the individual microdischarges were studied using digital storage oscilloscope and phaselocked ICCD imaging of the discharge pattern. The results were compared with numerical modelling of the electric field distribution around the discharge cell, and the results are given below.

The key studied parameter was the change in the operational voltage of DCSBD with increasing barrier thickness. Three different alumina ceramic plates were used with thicknesses of $0.2,0.6$ and $1.0 \mathrm{~mm}$, while the other parameters, e.g., as the geometry of the discharge cell and the composition of the electrodes (silver) and the dielectric support plate (glass-ceramic) were kept same. As can be seen from the prospective of simple electrostatic principles, the dielectric plate covering the electrodes will substantially influence the geometry and the intensity of the electric field generated around the electrodes. In the case of an alumina-to-air system, the electric field will tend to concentrate in the alumina plate and the electric field will be attenuated in the air gap above the plate. The electric field above the dielectric barrier will also get flattened with increasing dielectric barrier thickness, as the dielectric effectively shields the high gradients of the electric field generated by the electrodes.

For all experiments presented here, the discharge was first ignited and the power input was then maintained at a level of just complete plasma coverage of the DCSBD cell's surface at an operating frequency of $18 \mathrm{kHz}$. Figure 4 a shows the voltage waveforms for three dielectric barriers used in the experiments. Fig. $4 \mathrm{p}$ shows the corresponding current waveforms, only shifted by $100 \mathrm{~mA}$ up and down for better readability of the figure. The representative currentvoltage waveforms of DCSBD are given in Fig. 44. only for an alumina barrier $1.0 \mathrm{~mm}$ in thickness.
Fig. 4 a shows that the amplitude of the operating voltage increases considerably with the thickness of the dielectric barrier for the configuration of the discharge used in the experiments. The amplitude of current flow through the circuit increases as well, as the DCSBD is a capacitively coupled discharge, see Fig. 4 b. The current waveforms also indicate the influence of barrier thickness on the number of microdischarges per period. The thinner the barrier is, the higher number and the amplitude of the microdischarges that are seen on the current waveforms.

The high currents and voltages put stress on the power supply circuit and result in a considerable increase in power losses in the electric circuit and in the dielectrics. When the currents normed to excitation voltages are compared their amplitudes are practically the same for the barrier thickness used here. That imposes practically the same impedance of the discharge. This implies that the power to be delivered from the power supply rises with the second power of the input voltage amplitude. Higher excitation voltages also result in increased demands on the performance limits of the high voltage power supply. Higher excitation voltage values have to be considered in the structure of the secondary winding of the high-voltage transformer, where a higher dielectric-strength insulator has to be used between the primary and secondary windings, and also between the layers of the secondary windings. Higher amplitude of the electrical current flowing through the circuit necessitates the use of transformers made of conductors with a greater crosssection. Both factors have a negative influence on the transformer's coupling factor, which leads to higher voltage demands on the transistors of the power supply. The semiconductor switchers exciting the primary winding of the high-voltage transformer also have to be designed to accept higher amplitude of the current.

\subsection{FAST OPTICAL IMAGing}

ICCD images of the discharge patterns are given in Fig. 5 and Fig. 6 for alumina barrier thicknesses of 0.2 , $0.6 \mathrm{~mm}$ and $1.0 \mathrm{~mm}$. The individual microdischarges can easily be identified in the images of the whole single period of input voltage (Fig. 5), and also in 

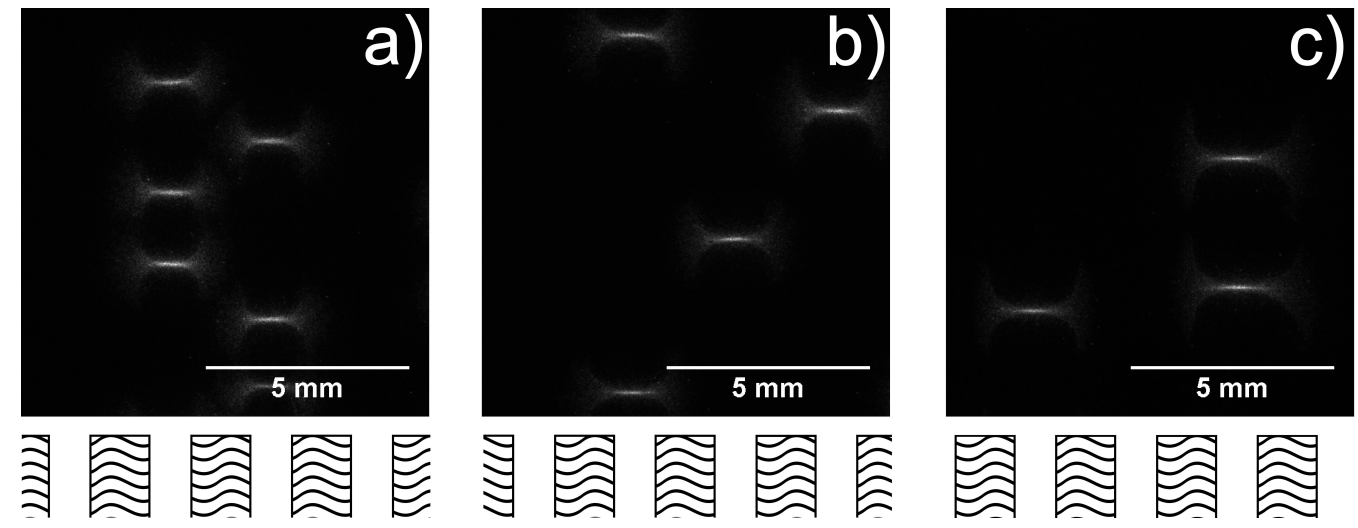

FiguRE 5. ICCD images of DCSBD generated in ambient air for various thicknesses of dielectric barrier - a) $0.2 \mathrm{~mm}$, b) $0.6 \mathrm{~mm}$ and c) $1.0 \mathrm{~mm}$. The images represent a single whole period of the discharge. The position of the electrodes beneath the dielectric barrier is given under each image.
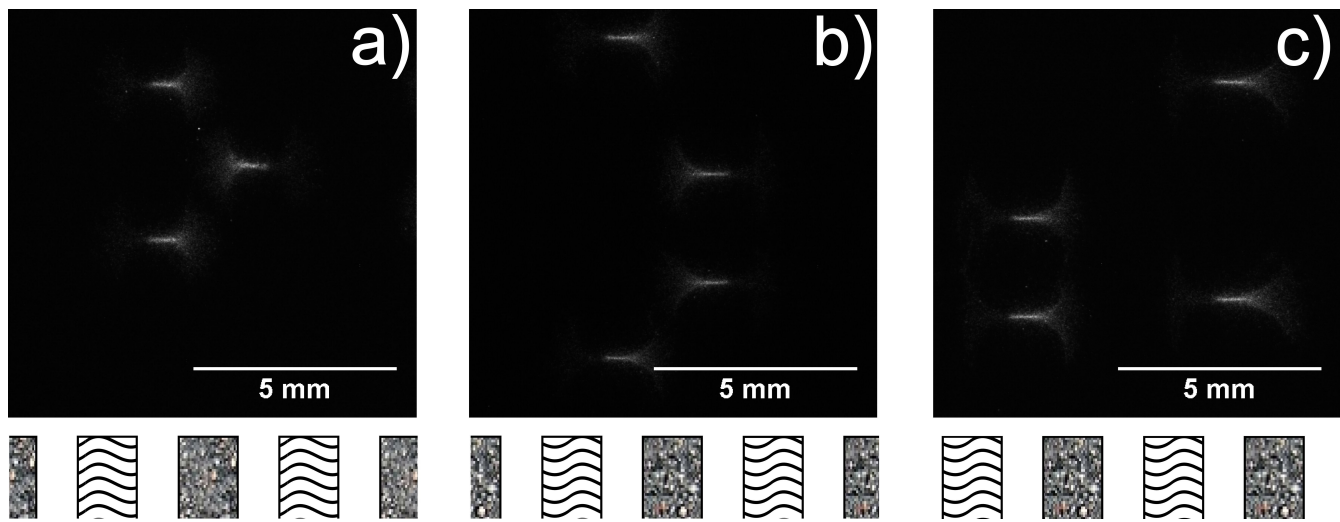

FiguRE 6. ICCD images of DCSBD generated in ambient air for various thicknesses of the dielectric barrier - a) $0.2 \mathrm{~mm}, \mathrm{~b}) 0.6 \mathrm{~mm}$ and c) $1.0 \mathrm{~mm}$. The images represent a single half-period of the discharge. The position of the electrodes beneath the dielectric barrier is given under each image. The actual cathodes are "wavy" patterned.

the images of half of a single period of input voltage (Fig. 6). The microdischarges have the most luminous parts connected to the anode oriented ionizing waves [20]. After the collision of the waves with the surface of the dielectric, branched surface streamers can be identified. Under given conditions, the individual microdischarges appear very similar for all barrier thicknesses, and an significant conclusion on the differences due to barrier thickness can not be drawn from the data. The length of the microdischarges is approx. $2.7 \mathrm{~mm}$, and is constant within the estimation error of approx. $0.2 \mathrm{~mm}$. The estimated diameter of the luminous channel of microdischarges is very close for all barrier thicknesses, ranging from $0.11 \pm 0.02 \mathrm{~mm}$ for a $0.2 \mathrm{~mm}$ thick barrier to $0.09 \pm 0.02 \mathrm{~mm}$ for a $1.0 \mathrm{~mm}$ thick barrier. The estimated optical resolution of our experimental setup is of the same order as the estimation error of the diameter. The maximum intensity of the microdischarges can be within the estimation error considered the same for all barrier thicknesses, ranging (in arbitrary units) from $9.0 \pm 1.5$ for a $0.2 \mathrm{~mm}$ thick barrier to $9.9 \pm 1.6$ for a $1.0 \mathrm{~mm}$ thick barrier.

\subsection{Electric Field Simulation}

A numerical simulation of the DCSBD cell's electrical field distribution was performed in order to better understand the experimentally obtained data and to show the influence of barrier thickness. A simplified model of the DCSBD cell was used to construct the $2 \mathrm{D}$ section of the DCSBD cell in the plane perpendicular to the surface of the dielectric barrier and electrodes. The computational domain is shown in Fig. 7. Four pairs of electrodes were used, embedded in dielectrics and covered with a dielectric barrier plate, all surrounded by air. A finite-element method (FEM) simulation of the static electric field distribution was performed using COMSOL Multiphysics 4.3b simulation and modelling software [28].

The idea of the simulation was to check and clarify the dependence of voltage on thickness measured for different barrier thicknesses. Our assumptions were that under complete plasma coverage of the surface of the barrier, the electric field intensities at the plasma layer should be parallel, as the ionization coefficients depend on the electric field intensity. To verify this assumption, the electric field distribution was simulated and the electric field intensity was probed at a position $0.3 \mathrm{~mm}$ above the dielectric barrier in the 

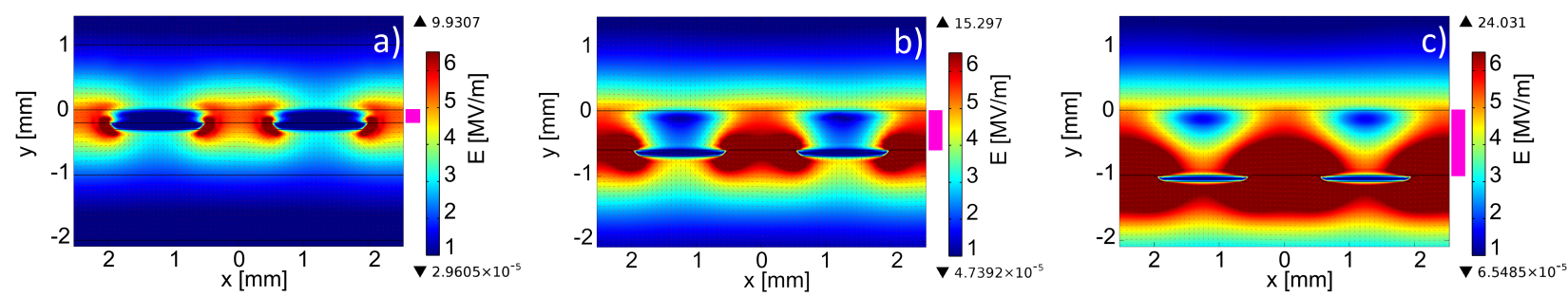

FIGURE 8. Detailed view of 2D electric field simulation around central electrode pair for different alumina thickness (denoted in figures as violet bar next to colorbar). Electric field intensities are given in $\mathrm{MV} \mathrm{m}^{-1}$ scale, minimum and maximum values of electric intensity in figure are also given. Arrows represents field vectors.

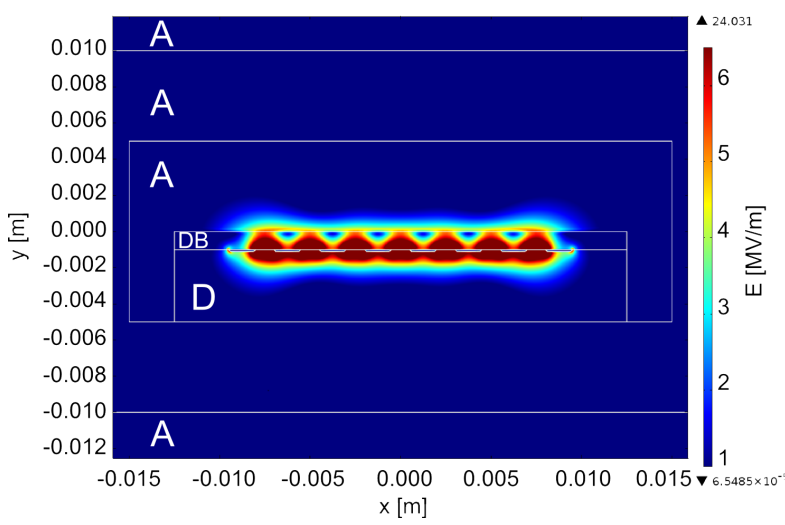

Figure 7. Computational domain of a $2 \mathrm{D}$ electric field simulation for thickness of alumina $1.0 \mathrm{~mm}$ and voltage amplitude of $14,9 \mathrm{kV}$. The electric field intensity is given in the $\mathrm{MV} \mathrm{m}^{-1}$ scale. Minimum and maximum values of the electric intensity are also given. (A) denotes air, (D) denotes dielectric, (DB) denotes dielectric barrier.

middle of the electrode gap (in the position of the plasma layer - see [19]). We set the initial conditions for the first simulation of the barrier $0.2 \mathrm{~mm}$ in thickness according to the measured operating voltage, i.e., $6.850 \mathrm{kV}$, and the electric field intensity in the given position was probed (reference intensity). Then the dielectric barrier thickness was increased in the model to values of 0.6 and $1.0 \mathrm{~mm}$, and the voltage of the electrodes was iteratively raised until close-to-reference electric field intensity was achieved in the probe position.

Fig. 8 shows the results of numerical simulations. The violet bars between the graph and the colorbar represent the position and the actual thickness of the dielectric barrier. The simulation confirms that keeping the electric field intensity at point $(0.3,0.3 \mathrm{~mm})$ above the dielectric requires the same voltage increase as shown in Fig. 4 (4). This point of interest represents the typical position of a streamer above the dielectric barrier in the middle of the discharge electrode configuration. Specifically, values of $6.9 \mathrm{kV}, 9.8 \mathrm{kV}$ and $14.9 \mathrm{kV}$ were obtained from the simulation using barrier thickness $0.2,0.6$ and $1.0 \mathrm{~mm}$. Despite the simplified model geometry and omission of the discharge dynamics the simulation data were in good agreement with the experimentally obtained data, that were $6.9,9.5$ and $14.9 \mathrm{kV}$ for barrier thickness $0.2,0.6$ and $1.0 \mathrm{~mm}$. We can conclude that the external electric field intensity in the plasma layer is one of the scaling parameters influencing DCSBD. When the dielectric barrier thickness is increased, an appropriate increase in operating voltage has to be made in order to achieve uniform/full plasma coverage of the dielectric barrier. Raising the operating voltage, on the other hand, puts more stress on the electric power supply circuit design, see the discussion in the previous section.

\section{Conclusions}

The effect of dielectric barrier thickness on DCSBD discharge properties has been shown. The barrier thickness influences the electrical parameters of the discharge, and also imposes the parameters of a highvoltage power supply unit. The thinner the dielectric barrier is, the less voltage amplitude is required to excite the discharge. This has practical impacts on the dielectric strength of dielectric embedding electrode system, and also on the transformer insulation, which is less stressed. In addition the current flow through the electric power circuit is weaker, enabling lightweight design of the high-voltage power supply unit. By contrast, the thinner the dielectric barrier is, the more fragile the whole discharge cell becomes. This greatly limits the practical usage of this kind of plasma system. According to our experience, a $0.6 \mathrm{~mm}$ thick alumina barrier provides balanced mechanical and electrical performance of the DCSBD discharge.

\section{ACKNOWLEDGEMENTS}

This research has been supported by the project $R \& D$ centre for low-cost plasma and nanotechnology surface modifications CZ.1.05/2.1.00/03.0086 funded by European Regional Development Fund.

\section{REFERENCES}

[1] W. Siemens. Ueber die elektrostatische Induction und die Verzögerung des Stroms in Flaschendrähten. Annalen der Physik und Chemie 178(9):66-122, 1857. DOI:10.1002/andp.18571780905

[2] U. Kogelschatz. Dielectric-Barrier Discharges: Their History, Discharge Physics, and Industrial Applications. Plasma chemistry and plasma processing 23(1):1-46, 2003. DOI:10.1023/A:1022470901385. 
[3] B. Eliasson, M. Hirth, U. Kogelschatz. Ozone synthesis from oxygen in dielectric barrier discharges. Journal of Physics D: Applied Physics 20(11):1421-1437, 1987. DOI:10.1088/0022-3727/20/11/010.

[4] C. Humpert, G. J. Pietsch. Simulation of Ozone Synthesis in Oxygen- and Air-Fed Surface Discharge Arrangements. Ozone: Science \& Engineering 27(1):59-68, 2005. DOI:10.1080/01919510590909048.

[5] K. H. Becker, U. Kogelschatz, K. H. Schoenbach, R. J. Barker (eds.). Non-Equilibrium Air Plasmas At Atmospheric Pressure. Institute of Physics Publishing, Bristol, 2004.

[6] U. Kogelschatz, B. Eliasson, W. Egli. Dielectric-Barrier Discharges. Principle and Applications. Le Journal de Physique IV 07(C4):C4-47-C4-66, 1997. DOI:10.1051/jp4:1997405

[7] M. Černák, L. Černáková, I. Hudec, et al. Diffuse Coplanar Surface Barrier Discharge and its applications for in-line processing of low-added-value materials. The European Physical Journal Applied Physics 47(2):22806, 2009. DOI:10.1051/epjap/2009131.

[8] C. Lux, Z. Szalay, W. Beikircher, et al. Investigation of the plasma effects on wood after activation by diffuse coplanar surface barrier discharge. European Journal of Wood and Wood Products 2013. DOI:10.1007/s00107-013-0706-3

[9] G. Borcia, C. A. Anderson, N. M. D. Brown. Dielectric barrier discharge for surface treatment: application to selected polymers in film and fibre form. Plasma Sources Science and Technology 12(3):335-344, 2003. DOI:10.1088/0963-0252/12/3/306

[10] P. Stahel, V. Buršíková, J. Buršík, et al. Hydrophylisation of non-woven polypropylene textiles using atmospheric pressure surface barrier discharge. Journal of Optoelectronics and Advanced Materials 10(1):213-218, 2008.

[11] K. D. Weltmann, E. Kindel, T. von Woedtke, et al. Atmospheric-pressure plasma sources: Prospective tools for plasma medicine. Pure and Applied Chemistry 82(6):1223-1237, 2010. DOI:10.1351/PAC-CON-09-10-35

[12] A. S. Wu, S. Kalghatgi, D. Dobrynin, et al. Porcine intact and wounded skin responses to atmospheric nonthermal plasma. The Journal of Surgical Research 179(1):e1-e12, 2013. DOI:10.1016/j.jss.2012.02.039.

[13] R. Brandenburg, J. Ehlbeck, M. Stieber, et al. Antimicrobial Treatment of Heat Sensitive Materials by Means of Atmospheric Pressure Rf-Driven Plasma Jet. Contributions to Plasma Physics 47(1-2):72-79, 2007. DOI:10.1002/ctpp.200710011

[14] J. Vrajova, L. Chalupova, O. Novotny, et al. Removal of paper microbial contamination by atmospheric pressure DBD discharge. The European Physical Journal D 54(2):233-237, 2009. DOI:10.1140/epjd/e2009-00127-4

[15] J. Reece Roth. Industrial Plasma Engineering. Taylor \& Francis, Bristol and Philadelphia, 2001. DOI:10.1201/9781420034127

[16] J. R. Roth, J. Rahel, X. Dai, D. M. Sherman. The physics and phenomenology of One Atmosphere Uniform Glow Discharge Plasma (OAUGDP ${ }^{\mathrm{TM}}$ ) reactors for surface treatment applications. Journal of Physics D: Applied Physics 38(4):555-567, 2005. DOI:10.1088/0022-3727/38/4/007.

[17] N. Gherardi, G. Gouda, E. Gat, et al. Transition from glow silent discharge to micro-discharges in nitrogen gas. Plasma Sources Science and Technology 9(3):340-346, 2000. DOI:10.1088/0963-0252/9/3/312

[18] N. Naudé, J.-P. Cambronne, N. Gherardi, F. Massines. Electrical model and analysis of the transition from an atmospheric pressure Townsend discharge to a filamentary discharge. Journal of Physics D: Applied Physics 38(4):530-538, 2005. DOI:10.1088/0022-3727/38/4/004

[19] M. Šimor, J. Ráhel', P. Vojtek, et al. Atmospheric-pressure diffuse coplanar surface discharge for surface treatments. Applied Physics Letters 81(15):2716, 2002. DOI:10.1063/1.1513185

[20] T. Hoder, R. Brandenburg, R. Basner, et al. A comparative study of three different types of barrier discharges in air at atmospheric pressure by cross-correlation spectroscopy. Journal of Physics D: Applied Physics 43(12):124009, 2010. DOI:10.1088/0022-3727/43/12/124009

[21] M. Černák, D. Kováčik, J. Ráhel', et al. Generation of a high-density highly non-equilibrium air plasma for high-speed large-area flat surface processing. Plasma Physics and Controlled Fusion 53(12):124031, 2011. DOI:10.1088/0741-3335/53/12/124031.

[22] D. Korzec, E. Finantu-Dinu, A. Schwabedissen, et al. Insulated surface discharge for metastables driven processing at atmospheric pressure. Surface and Coatings Technology 169-170:228-232, 2003. DOI:10.1016/S0257-8972(03)00086-0

[23] M. Štefečka, M. Kando, M. Černák, et al. Spatial distribution of surface treatment efficiency in coplanar barrier discharge operated with oxygen-nitrogen gas mixtures. Surface and Coatings Technology 174175:553-558, 2003. DOI:10.1016/S0257-8972(03)00332-3.

[24] J. Čech, P. Stahel, Z. Navrátil. The influence of electrode gap width on plasma properties of diffuse coplanar surface barrier discharge in nitrogen. The European Physical Journal D 54(2):259-264, 2009. DOI:10.1140/epjd/e2009-00013-1

[25] V. Prysiazhnyi, P. Slavicek, M. Cernak. Aging of plasma-activated copper and gold surfaces and its hydrophilic recovery after water immersion. Thin Solid Films 550:373-380, 2014. DOI:10.1016/j.tsf.2013.10.176

[26] V. Prysiazhnyi, A. Brablec, J. Čech, et al. Generation of Large-Area Highly-Nonequlibrium Plasma in Pure Hydrogen at Atmospheric Pressure. Contributions to Plasma Physics 54(2):138-144, 2014. DOI:10.1002/ctpp.201310060

[27] T. Homola, J. Matoušek, V. Medvecká, et al. Atmospheric pressure diffuse plasma in ambient air for ITO surface cleaning. Applied Surface Science 258(18):7135-7139, 2012. DOI:10.1016/j.apsusc.2012.03.188

[28] COMSOL Multiphysics ${ }^{\circledR}$ software version $4.3 \mathrm{~b}$ COMSOL, Inc., Burlington, MA, USA, 2013. http://www.comsol.com [2014-12-01] 